\title{
The Impact Of Hyperuricemia On Cardiometabolic Risk Factors In Patients With Diabetes Mellitus: A Cross-Sectional Study
}

This article was published in the following Dove Press journal: Diabetes, Metabolic Syndrome and Obesity: Targets and Therapy

\author{
Laura Gaita' \\ Romulus Timar ${ }^{1,2}$ \\ Nicoleta Lupascu' \\ Deiana Roman (ID) \\ Alin Albai ${ }^{1,2}$ \\ Ovidiu Potre 3,4 \\ Bogdan Timar $\mathbb{D}^{2,5}$ \\ 'Second Department of Internal \\ Medicine, "Victor Babes" University of \\ Medicine and Pharmacy, Timisoara, \\ Romania; 'Department of Diabetes and \\ Metabolic Diseases, "Pius Brinzeu" \\ Emergency Hospital, Timisoara, Romania; \\ ${ }^{3}$ First Department of Internal Medicine, \\ "Victor Babes" University of Medicine \\ and Pharmacy, Timisoara, Romania; \\ ${ }^{4}$ Department of Hematology, Municipal \\ Emergency Hospital, Timisoara, Romania; \\ ${ }^{5}$ Department of Functional Sciences, \\ "Victor Babes" University of Medicine \\ and Pharmacy, Timisoara, Romania
}

Correspondence: Romulus Timar Second Department of Internal Medicine, "Victor Babes" University of Medicine and Pharmacy, 2 Eftimie Murgu, Timisoara 30004I, Romania

Tel +40748331 294

Email timar.romulus@umft.ro
Purpose: Hyperuricemia (HUA) is linked to a variety of non-communicable diseases such as atherosclerotic cardiovascular disease (ASCVD), chronic kidney disease (CKD) and hypertension, with evidence showing its role in the development of diabetes mellitus (DM). Our study's main aim was to explore the associations of HUA with other traditional risk factors in Romanian patients with $\mathrm{DM}$ and to assess the impact of the increase of serum UA on DM complications and HbAlc.

Patients and methods: In this cross-sectional, non-interventional study, we enrolled, according to a population-based, consecutive-case principle, 133 patients previously diagnosed with DM. HbA1c, uric acid, lipid profile, urinary albumin/creatinine ratio, glomerular filtration rate, $\mathrm{TSH}$ and $\mathrm{FT}_{4}$ measurements were performed, while the diagnosis of retinopathy and of diabetic neuropathy was established using standardized methods.

Results: An increased uric acid level was associated with a significant increase in the risk for development of stroke $(\mathrm{OR}=1.526 ; \mathrm{p}=0.004)$. A weak, positive and statistically significant correlation can also be observed between the BMI and the presence of hyperuricemia $(\mathrm{r}=0.131 ; \mathrm{p}=0.034)$, and between the triglyceride levels and hyperuricemia $(\mathrm{r}=0.173$; $\mathrm{p}=0.004$ ). Glomerular filtration rate was correlated to hyperuricemia in a strong and negative manner, having an important statistical significance $(\mathrm{r}=-0.818 ; \mathrm{p}=0.003)$. In our study, UA levels and $\mathrm{HbAlc}$ were negatively correlated, without reaching statistical significance.

Conclusion: Serum UA is strongly correlated with the BMI, triglyceride level and GFR in Romanian patients with DM and HUA is significantly associated with a higher risk of stroke in these individuals.

Keywords: uric acid, diabetes mellitus, metabolic syndrome, chronic diseases

\section{Introduction}

Diabetes mellitus (DM) is affecting almost half a billion people worldwide and its prevalence is rising continuously, being estimated to reach more than 620 million patients by 2045. It is also known that DM is one of the leading causes of cardiovascular disease (CVD), chronic kidney disease (CKD), vision loss and nontraumatic lower-limb amputation. ${ }^{1}$ Its presence is strongly associated with an increase in the incidence of both stable and unstable angina, non-fatal myocardial infarction, heart failure and peripheral arterial disease, ${ }^{2}$ doubling or even tripling the risk of developing atherosclerotic cardiovascular disease (ASCVD), the leading cause of mortality in the world. ${ }^{3}$

There is a growing body of evidence supporting the fact that high levels of uric acid (UA) are linked to multiple chronic diseases such as hypertension, chronic 
kidney disease, ASCVD, dyslipidemia and non-alcoholic fatty liver disease (NAFLD), ${ }^{4}$ while normal concentrations may have a protective effect against dementia (in those without cardiovascular risk factors) ${ }^{5}$ and on the bone metabolism. ${ }^{6}$

Emerging evidence underlines the role hyperuricemia (HUA) has in the development of DM, data from the Atherosclerosis Risk in Communities Study showing that UA levels are associated with an increased risk of DM even after adjustment for other risk factors, alongside findings from the Rotterdam Study that state that serum UA is a strong independent risk factor for DM and the Framingham Heart Study that proves that individuals with higher UA levels associate a greater risk for the development of T2DM. ${ }^{7-9}$ However, these studies relied on a singular measurement of serum UA levels, whereas it has been proven that persistent HUA is more appropriate in assessing the risk of DM development. ${ }^{10}$

Diabetic dyslipidemia is a cluster of plasma lipid and lipoprotein abnormalities and includes, among others, elevated triglycerides, low HDL cholesterol and an increase in small and dense LDL cholesterol. ${ }^{11,12}$ Increased production of triglycerides in the liver also associates with de novo purine synthetization, an accelerator of the production of UA. ${ }^{13}$

Diabetic retinopathy represents the most common cause of vision loss in patients with both T1DM and T2DM patients. In addition to insufficient glycemic control, recent studies found that high monosodium urate levels are correlated with increased inflammation markers and xanthine oxidase expression in the post-mortem analysis of diabetic patient's retinas and vitreous and concludes that even asymptomatic HUA is to be considered a risk factor for the induction and progression of diabetic retinopathy. ${ }^{14}$

It is common for patients with DM to have associated also several other risk factors, such as dyslipidemia, obesity and hypertension and multiple studies have proven the efficacy of addressing all of these conditions simultaneously. ${ }^{15} \mathrm{DM}$ is also included in the constellation that is represented by the metabolic syndrome (MS).

A singular definition for metabolic syndrome is not available due to slightly different criteria proposed by each of the working groups, thus leading to difficulties in establishing its prevalence. ${ }^{16}$ In spite of this aspect, there are elements of the MS cluster unanimously agreed upon, such as: DM and prediabetes, high blood pressure, elevated triglycerides and abdominal obesity. MS also includes a prothrombotic, hypofibrinolytic state, endothelial dysfunction, chronic subclinical inflammation, NAFLD, hemodynamic changes and HUA. ${ }^{17}$

Diabetic patients usually exhibit higher waist circumferences and higher BMIs, leading to higher leptin production and insulin resistance (IR), both of which are known to decrease the excretion rate of uric acid. ${ }^{13}$

The vast majority of cardiovascular events are caused by atherosclerosis, DM and MS being well known to accelerate this process. ${ }^{18}$ HUA is in itself a cause of endothelial dysfunction, therefore increasing the oxidative stress which leads to a decrease in nitric oxide bioavailability and vascular smooth muscle cell proliferation. ${ }^{19}$

A higher risk of premature death was found in patients with gout, as shown from results of large-scale epidemiological studies. ${ }^{20,21}$ Men with gout had a $28 \%$ higher risk of death compared to men without gout, independent from other risk factors. ${ }^{22}$ Associating both the diagnosis of gout and that of HUA increases risk of all-cause mortality mainly derived from an increased CVD mortality. ${ }^{20}$

Given the fact that HUA, CVD and DM oftentimes associate in a patient, special attention must be provided to the treatment of these individuals in order to lower mortality and complication rates, as well as to offer the best quality of life. One study showed that the administration of urate-lowering therapy, namely allopurinol, led to a $23 \%$ decrease in mortality, although researchers are unsure if this effect was solely due to the lowering of urate levels or to other beneficial effects of the drug. These results were obtained independent from other factors such as age, sex, BMI, albumin, GFR, comorbidities or health-care utilisation. ${ }^{23}$ It is even suggested that an effective control of serum UA may have an important role in the prevention of CVD. ${ }^{24}$

Having these premises, the aim of our study was to explore the associations of HUA with other traditional risk factors in Romanian patients with DM and to assess the impact of the increase of serum UA on DM complications and on $\mathrm{HbAl}$.

\section{Patients And Methods \\ Study Design And Patients}

In this cross-sectional, non-interventional study, we enrolled 133 patients previously diagnosed with DM, admitted in the Diabetes Compartment of the Emergency Hospital Timisoara, Romania. Patients participating in the study were enrolled according to a population-based, consecutive-case principle. All patients provided written 
informed consent prior to any study procedure or activity. Since this is a non-interventional, cross-sectional study, according to the local protocols, it had to be approved only by the Ethics Committee of the "Pius Brinzeu" Emergency Hospital Timisoara. The entire study was conducted according to the principles stated in the Declaration of Helsinki. Exclusion criteria had been considered to be the inability to provide informed consent, the inability to provide accurate anamnestic data or any condition which, in the investigators' opinion, could lead to biases in the study results.

\section{Clinical, Anthropometric And Laboratory Data}

Data regarding the patients' age, body mass index (BMI), hypertension, coronary artery disease, stroke, peripheral artery disease and peripheral venous insufficiency were collected from the patients' medical records. HbA1c, $\mathrm{UA}$, lipid profile, urinary albumin/creatinine ratio, glomerular filtration rate, $\mathrm{TSH}$ and $\mathrm{FT}_{4}$ measurements were performed after at least $12 \mathrm{hrs}$ of fasting and the drawn blood and the collected urine were analyzed using standardized methods. UA was measured once during the study. We considered as HUA levels that exceeded the UA reference values of our laboratory which are $2.6-6 \mathrm{mg} / \mathrm{dL}$ in women and $3.5-7.2 \mathrm{mg} / \mathrm{dL}$ in men. The diagnosis of retinopathy was established after performing a fundoscopic examination by the same trained ophthalmologist, specialized in the diabetic eye complications. The presence of diabetic neuropathy was assessed using the 10-g monofilament test, pinprick and temperature sensation test and vibration perception test using a $128-\mathrm{Hz}$ tuning fork. The diagnosis of stroke was based on history taking, while the diagnosis of peripheral artery disease was established using the anklebrachial index.

\section{Statistical Analysis}

Data were collected and analyzed using SPSS v.17 (SPSS Inc, Chicago, IL, USA). The results are presented as mean \pm standard deviation for numerical variables with Gaussian distribution, median and [interquartile range] for numerical variables with non-parametric distribution, respectively, as percentage from the subgroup's total and frequencies for nominal variables. To assess the normality of the numerical variable's distribution, the Shapiro and Wilk method was used - a p-value lower than 0.05 obtained at this test was associated with non-parametric distributions.
The statistical significance of differences between groups was assessed using the unpaired t-student test (numerical variables with Gaussian distribution), MannWhitney $U$-test (numerical variables with non-parametric distribution) respectively, the Chi-Square test for nominal variables.

To evaluate the impact of the UA levels on the risk for development of several pathologies and complications univariable and multivariable logistic regression models were built, containing the UA levels as a predictor. The selection of the other predictors in the multivariable regression models was performed in a backward-stepwise manner.

The sample size of the studied lot was calculated prior enrollment, based on previous similar literature data, to provide a confidence level of $95 \%$ and a statistical power of at least $80 \%$.

In this study, a p-value lower than 0.05 was considered the threshold of statistical significance.

\section{Results}

Our study lot included 133 patients of which 53.4\% (71) were male. The median age of these patients was 63 [54-69]. The prevalence of patients with hyperuricemia was $26.3 \%$ (35 cases), 66.2\% (88) had diabetic neuropathy, 27.1\% (36) suffered from diabetic retinopathy, 81.2\% (108) had hypertension, $60.2 \%$ (80) were known to have coronary artery disease, $8.3 \%$ (11) presented with a history of stroke, peripheral artery disease was encountered in 9.8\% (13) of patients and peripheral venous insufficiency in $21.1 \%$ (28). A detailed rendition of the patient's general characteristics at enrolment can be found in Table 1 .

The presence of hyperuricemia was associated with a decreased value of estimated glomerular filtration rate (65 $\mathrm{mL} / \mathrm{min}$ vs $77.5 \mathrm{~mL} / \mathrm{min}$; $\mathrm{p}=0.011$; Figure 1). This association and other parameters stratified according to the presence of hyperuricemia can be found in Table 2 .

Of the patients with HUA, our data show that 15 $(42.9 \%)$ were male and $20(57.1 \%)$ were female, gender being not statistically significant associated to the development of this pathology $(\mathrm{p}=0.146)$.

Hyperuricemia was present in $5(14.3 \%)$ of those without clinical obesity, in $6(17.1 \%)$ of the overweight patients, and in $24(68.6 \%)$ of those with obesity, without statistical significance $(\mathrm{p}=0.183)$.

Although our data could not statistically link diabetic neuropathy to hyperuricemia $(p=0.726), 24(68.6 \%)$ of those with hyperuricemia also presented with DN. 
Table I Patients' General Characteristics At Enrolment

\begin{tabular}{|c|c|}
\hline Age $(\text { years })^{a}$ & $63[54-69]$ \\
\hline Male gender ${ }^{b}$ & 71 (53.4\%) \\
\hline $\mathrm{HbAlc}(\%)^{\mathrm{c}}$ & $8.9 \pm 1.8$ \\
\hline BMI $\left(\mathrm{kg} / \mathrm{m}^{2}\right)^{\mathrm{c}}$ & $32.2 \pm 6.3$ \\
\hline Hyperuricemia (\%) & $35(26.3 \%)$ \\
\hline Diabetic neuropathy (\%) ${ }^{\mathrm{b}}$ & $88(66.2 \%)$ \\
\hline Diabetic retinopathy $(\%)^{\mathrm{b}}$ & $36(27.1 \%)$ \\
\hline Hypertension (\%) & $108(81.2 \%)$ \\
\hline Coronary artery disease (\%) & $80(60.2 \%)$ \\
\hline History of stroke (\%) ${ }^{\mathrm{b}}$ & II (8.3\%) \\
\hline Peripheral artery disease $(\%)^{\mathrm{b}}$ & $13(9.8 \%)$ \\
\hline Peripheral venous insufficiency $(\%)^{b}$ & $28(21.1 \%)$ \\
\hline Total cholesterol $(\mathrm{mg} / \mathrm{dL})^{c}$ & $199.2 \pm 85.1$ \\
\hline $\operatorname{LDLc}(\mathrm{mg} / \mathrm{dL})^{\mathrm{c}}$ & $113.4 \pm 50.8$ \\
\hline $\operatorname{HDLc}(\mathrm{mg} / \mathrm{dL})^{\mathrm{c}}$ & $41.6 \pm 13.9$ \\
\hline Triglycerides $(\mathrm{mg} / \mathrm{dL})^{\mathrm{a}}$ & $149[102-256]$ \\
\hline Dyslipidemia (\%) ${ }^{\mathrm{b}}$ & $87(65.4 \%)$ \\
\hline Uric acid $(\mathrm{mg} / \mathrm{dL})^{\mathrm{c}}$ & $5.6 \pm 2$ \\
\hline Urinary albumin/creatinine ratio $(\mathrm{mg} / \mathrm{g})^{\mathrm{a}}$ & $27.7[13-77]$ \\
\hline Glomerular filtration rate $\left(\mathrm{mL} / \mathrm{min} / 1.73 \mathrm{~m}^{2}\right)^{\mathrm{a}}$ & $78[58-91]$ \\
\hline $\mathrm{TSH}(\mathrm{mU} / \mathrm{L})^{\mathrm{a}}$ & $1.85[1.1-3.04]$ \\
\hline $\mathrm{FT}_{4}(\mathrm{ng} / \mathrm{L})^{\mathrm{c}}$ & $14.89 \pm 4.6$ \\
\hline
\end{tabular}

Notes: ${ }^{a}$ Numerical variables with non-parametric distribution. Results are presented as median and [interquartile range]. ${ }^{b}$ Categorical variables. Results are presented as number of cases and (percentage from the total). ${ }^{c}$ Numerical variables with Gaussian distribution. Results are presented as mean \pm standard deviation.

Of all patients with hyperuricemia, only 9 (25.7\%) also had diabetic retinopathy, a statistical significance between the two not being reached $(\mathrm{p}=0.834)$.

$30(85.7 \%)$ of those with hyperuricemia also presented hypertension, without any statistical significance attributed to the link between the two $(p=0.426)$.

Coronary artery disease patients represented a total of $23(65.7 \%)$ within the hyperuricemic lot, with a value of $\mathrm{p}=0.433$, inferring statistical significance.

$7(20 \%)$ of patients with hyperuricemia had a history of stroke, this association having a statistically significant quality $(\mathrm{p}=0.003)$.

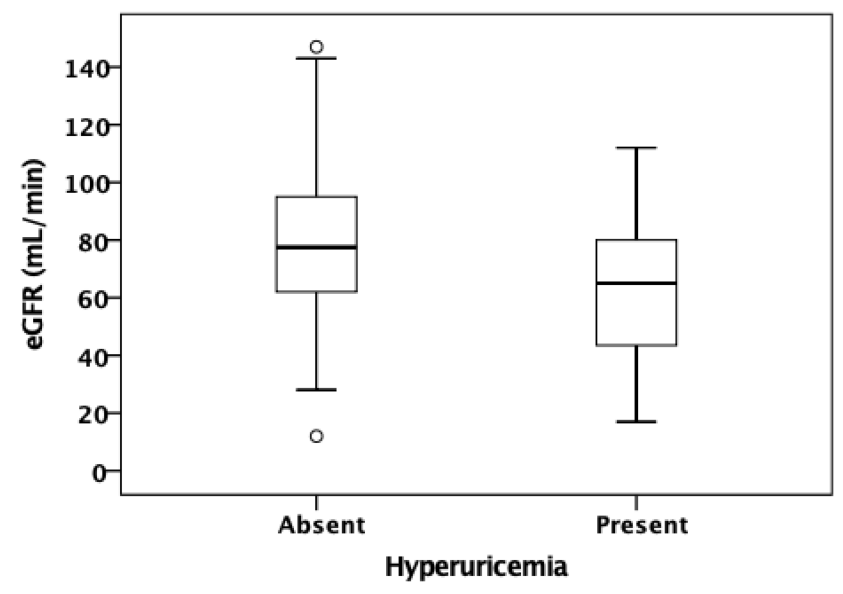

Figure I eGFR values in patient with vs without hyperuricemia.

$8(22.9 \%)$ of the hyperuricemic patients had chronic venous insufficiency, however without a statistical significance $(\mathrm{p}=0.760)$.

Steatohepatitis was present in 18 (51.4\%) of those with hyperuricemia, although in spite of this important number, a statistical significance regarding the two conditions could not be established $(\mathrm{p}=0.780)$.

A summary of the data presented above can be found in Table 3.

A weak, positive and statistically significant correlation can be observed between the patient's BMI and the presence

Table 2 Patients' Characteristics Stratified According To The Presence Of Hyperuricemia

\begin{tabular}{|c|c|c|c|}
\hline & $\begin{array}{l}\text { Without } \\
\text { Hyperuricemia }\end{array}$ & $\begin{array}{l}\text { Hyperuricemia } \\
\text { Present }\end{array}$ & $\mathbf{p}$ \\
\hline Age $(\text { years })^{b}$ & 62 [54-67] & 64 [54-70] & 0.412 \\
\hline $\mathrm{HbAlc}(\%)^{\mathrm{a}}$ & $8.9 \pm 1.6$ & $8.7 \pm 2.3$ & 0.659 \\
\hline BMI $\left(\mathrm{kg} / \mathrm{m}^{2}\right)^{\mathrm{a}}$ & $31.5 \pm 5.4$ & $33.5 \pm 8.3$ & 0.168 \\
\hline $\mathrm{TC}(\mathrm{mg} / \mathrm{dL})^{\mathrm{a}}$ & $197 \pm 92$ & $205 \pm 64$ & 0.634 \\
\hline $\mathrm{LDL}_{\mathrm{c}}(\mathrm{mg} / \mathrm{dL})^{\mathrm{a}}$ & $113.0 \pm 53.6$ & $1 \mid 4.7 \pm 42.9$ & 0.866 \\
\hline $\mathrm{HDL}_{\mathrm{c}}(\mathrm{mg} / \mathrm{dL})^{\mathrm{a}}$ & $42.7 \pm 14.2$ & $38.5 \pm 12.5$ & 0.122 \\
\hline TG $(\mathrm{mg} / \mathrm{dL})^{\mathrm{b}}$ & $14 \mid$ [97-248] & 176 [125-282] & 0.81 \\
\hline 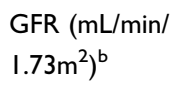 & 77.5 [62-95] & 65 [43-82] & $0.011 *$ \\
\hline ACR $(\mathrm{mg} / \mathrm{g})^{\mathrm{b}}$ & 27 [12.9-79.5] & $30.3[13-68]$ & 0.85 \\
\hline $\mathrm{TSH}(\mathrm{mU} / \mathrm{L})^{\mathrm{b}}$ & $1.85[1.2-2.9]$ & $1.9[0.9-3.4]$ & 0.792 \\
\hline $\mathrm{FT}_{4}(\mathrm{ng} / \mathrm{L})^{\mathrm{a}}$ & $14.57 \pm 4.37$ & $15.81 \pm 5.15$ & 0.298 \\
\hline
\end{tabular}

Notes: *Differences are statistically significant at $\alpha<0.05$ threshold. ${ }^{\text {a Numerical }}$ variables with Gaussian distribution. Results are presented as average \pm standard deviation. $p$ value was calculated using unpaired t-student test. ${ }^{b}$ Numerical variables with non-parametric distribution. Results are presented as median and [interquartile range]. $P$ value was calculated using Mann-Whitney $U$-test.

Abbreviations: BMI, body mass index; TC, total cholesterol; ACR, urinary albumin/creatinine ratio; TSH, Thyroid-stimulating hormone; $\mathrm{FT}_{4}$, Thyroxine. 
Table 3 Hyperuricemic Patient's Associated Pathologies

\begin{tabular}{|l|l|l|}
\hline & Hyperuricemia & P value \\
\hline Diabetic neuropathy (\%) & $24(68.6 \%)$ & 0.726 \\
Diabetic retinopathy (\%) & $9(25.7 \%)$ & 0.834 \\
Hypertension (\%) & $30(85.7 \%)$ & 0.426 \\
Coronary artery disease (\%) & $23(65.7 \%)$ & 0.433 \\
History of stroke (\%) & $7(20 \%)$ & $0.003 *$ \\
Chronic venous insufficiency (\%) & $8(22.9 \%)$ & 0.760 \\
Steatohepatitis (\%) & $18(51.4 \%)$ & 0.780 \\
Obesity & & \\
Normal BMI (\%) & $5(14.3 \%)$ & \\
Overweight (\%) & $6(17.1 \%)$ & 0.183 \\
Obese (\%) & $24(68.6)$ & \\
Gender & & \\
Male & $15(42.9 \%)$ & \\
Female & $20(57.1 \%)$ & 0.146 \\
\hline
\end{tabular}

Notes: *Differences are statistically significant at $\alpha<0.05$ threshold. Categorical variables. Results are presented as number of cases and (percentage from the total).

of hyperuricemia ( $\mathrm{r}=0.131 ; \mathrm{p}=0.034$; Figure 2$)$, suggesting that hyperuricemia is more likely to appear with an increase in body weight.

Triglyceride levels and hyperuricemia were also found to be weakly and positively correlated, their correlation being statistically significant $(r=0.173 ; p=0.004)$, implying that higher triglyceride levels could be found in patients presenting with hyperuricemia.

Glomerular filtration rate was correlated to hyperuricemia in a strong and negative manner, having an important statistical significance $(\mathrm{r}=-0.818 ; \mathrm{p}=0.003$; Figure 3$)$, indicating that hyperuricemia appears in patients with lower glomerular filtration rates.

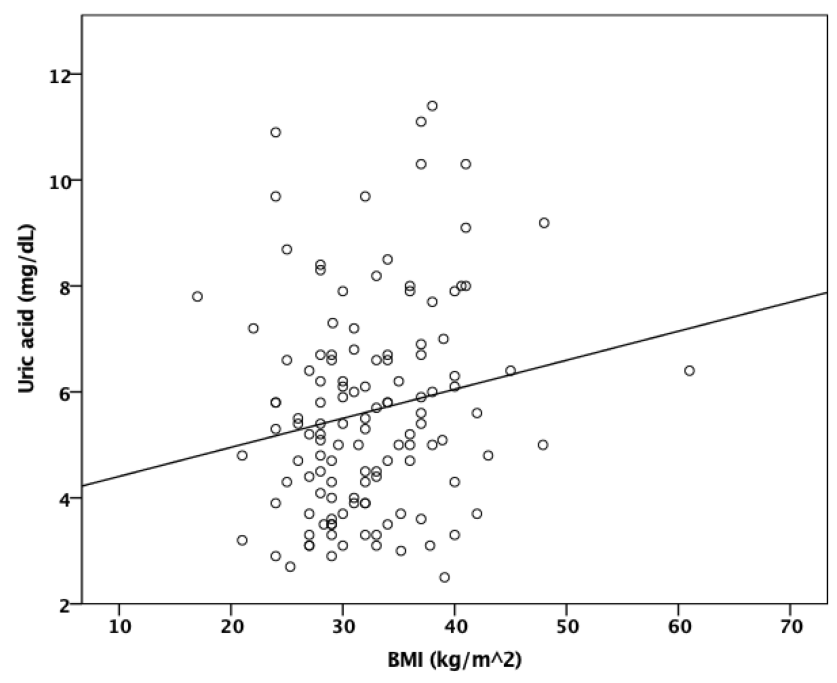

Figure 2 Correlation between body mass index and uric acid levels.

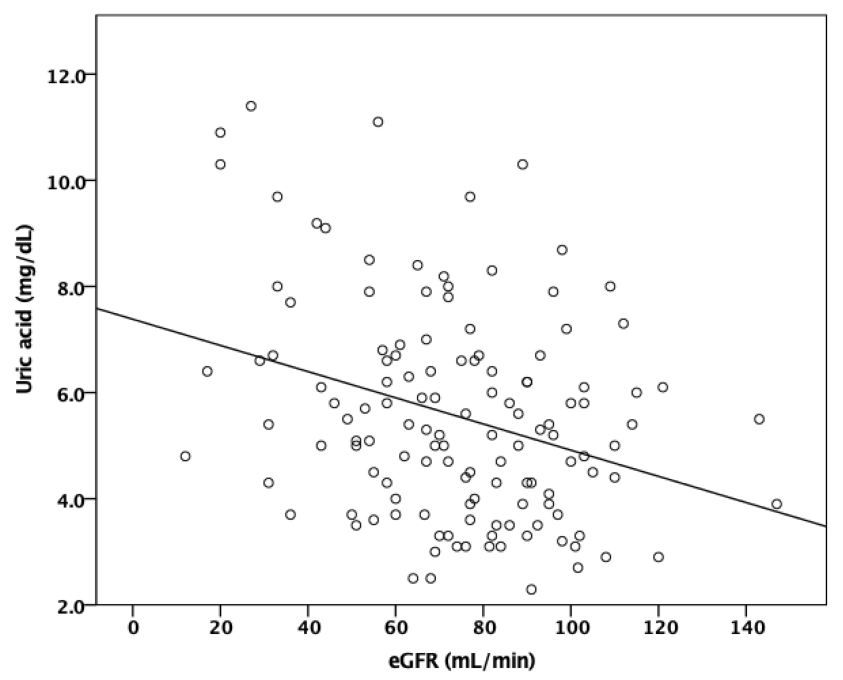

Figure 3 Correlation between estimated glomerular filtration rate and uric acid levels.

The detailed correlation analysis between uric acid levels and several parameters is presented in Table 4.

In the univariate logistic regression models built, the UA levels were not associated with a significant impact on the risk for development of diabetic neuropathy $(\mathrm{OR}=1.005$; $\mathrm{p}=0.956)$, diabetic retinopathy $(\mathrm{OR}=0.936 ; \mathrm{p}=0.936)$, hypertension $(\mathrm{OR}=1.301 ; \mathrm{p}=0.053)$ or coronary artery disease $(\mathrm{OR}=1.066 ; \mathrm{p}=0.489)$.

An increased uric acid level was associated with a significant increase in the risk for development of stroke $(\mathrm{OR}=1.526 ; \mathrm{p}=0.004)$. This increase in risk was observed also after correcting several confounding factors, like LDLc, BMI and presence of hypertension (Table 5).

Table 4 Correlation Between Patient's Characteristics And Uric Acid Values

\begin{tabular}{|l|l|l|}
\hline & Uric Acid & P value \\
\hline Age & 0.048 & 0.431 \\
Ketoacidosis & 0.064 & 0.314 \\
BMI & 0.131 & $0.034^{*}$ \\
Total Cholesterol & 0.052 & 0.383 \\
LDL $_{c}$ & 0.036 & 0.545 \\
$\mathrm{HDL}_{\mathrm{c}}$ & -0.107 & 0.077 \\
$\mathrm{TG}$ & 0.173 & $0.004^{*}$ \\
$\mathrm{HbAlc}$ & -0.096 & 0.110 \\
$\mathrm{GFR}$ & -0.818 & $0.003^{*}$ \\
$\mathrm{ACR}$ & 0.088 & 0.151 \\
$\mathrm{TSH}$ & -0.001 & 0.982 \\
$\mathrm{FT}_{4}$ & -0.010 & 0.890 \\
\hline
\end{tabular}

Notes: Spearman correlation coefficient. *Differences are statistically significant at $\alpha<0.05$ threshold. 
Table 5 Multivariate Regression Model, Analysing The Risk Of Stroke

\begin{tabular}{|l|l|l|l|}
\hline Parameter & OR & $\mathbf{9 5 \%} \mathbf{C l}$ & p-value \\
\hline Uric acid $(\mathrm{mg} / \mathrm{dL})$ & 1.647 & 1.163 to 2.332 & 0.005 \\
BMI $\left(\mathrm{kg} / \mathrm{m}^{2}\right)$ & 0.936 & 0.827 to 1.058 & 0.291 \\
LDLc $(\mathrm{mg} / \mathrm{dL})$ & 0.990 & 0.975 to 1.005 & 0.192 \\
\hline
\end{tabular}

\section{Discussion}

\section{Findings And Interpretation}

This study showed that serum UA is significantly correlated with the BMI, triglyceride level and glomerular filtration rate (GFR) and that HUA is associated with a higher risk of stroke, all of the above in diabetic patients.

Regarding the weight, it is already known that serum UA predicts future weight gain and that HUA induces oxidative stress in the adipose tissue with an impact on insulin resistance and, subsequently, on ASCVD and while it is proven that UA correlates better and positively with waist circumference that with the $\mathrm{BMI},{ }^{25}$ the results of our study are similar with the ones already described in literature.

This study's findings about serum UA and triglycerides are supported by others that have confirmed a strong correlation between the dyslipidemia characteristic for metabolic syndrome and UA levels - even better than with the parameters of insulin resistance. ${ }^{13}$

HUA is very prevalent in patients with chronic kidney disease. Moreover, several studies have reported an association between HUA and the reduction of GFR in patients with or without DM, some of them with conflicting results. ${ }^{26}$ In our study, serum UA is strongly and negatively correlated with the estimated GFR.

Although multiple studies have shown that HUA is a risk factor for diabetic retinopathy, ${ }^{14}$ our data could not statistically link the high levels of UA to this complication. The same applies in our lot of diabetic patients to an association between HUA and hypertension, HUA and coronary artery disease (CAD) and HUA and NAFLD.

However, studies that have investigated an association between serum UA and stroke, in diabetic and non-diabetic patients had conflicting results, some showing a significant and positive correlation, while others concluded the opposite. ${ }^{27} \mathrm{~A}$ meta-analysis including patients with type $2 \mathrm{DM}$ revealed that increased levels of UA may contribute to the development of an ischemic stroke, other prospective studies being recommended. ${ }^{28}$ In our study, a statistical significance regarding the association between HUA and a history of stroke was established.

In regard to the interaction between UA levels and glycaemic values, results vary from suggesting that UA is associated with $\mathrm{HbAlc}$ independently of other metabolic factors, ${ }^{29}$ to highlighting a non-linear relation between UA and blood glucose levels ${ }^{30}$ to some concluding that UA levels are negatively correlated with $\mathrm{HbA} 1 \mathrm{c}$ in patients with type 2 DM and positively correlated in individuals with a normal glucose metabolism. ${ }^{31}$ The results of our study show that in diabetic patients UA levels and $\mathrm{HbA} 1 \mathrm{c}$ are negatively correlated, without reaching statistical significance.

However, our patients were already following an antidiabetic drug regimen and, in some cases, a hypouricemic therapy. Recent studies have investigated the impact of allopurinol on multiple conditions - results suggesting that it lowers the incidence of ischemic stroke, ${ }^{32}$ conflicting results occurring while investigating whether it prevents vascular complications in diabetic patients, ${ }^{33}$ findings that could interfere with our data.

Some of the patients included in our study were following an antidiabetic regimen that included a sodium-glucose cotransporter 2 inhibitor, agents that were proven to lower the UA levels ${ }^{34}$ and consequently possibly leading to underestimating the prevalence and impact of HUA in diabetic patients.

\section{Strengths And Weaknesses Of The Study}

The strengths of the study are represented by the patients' consecutive enrolment principle which provided a study cohort including individuals with and without hyperuricemia, with a heterogenous age, BMI, glycaemic control, lipid and complication profile. The sample size is another strong point of our study design, providing enough statistical power and allowing the inference of the results obtained in the sample for the population of interest.

One of the weak points of the study is represented by the possible discrepancy between the characteristics of the evaluated patients and of the general population with DM caused by enrolling individuals admitted in the Diabetes Compartment of the Emergency Hospital for various symptoms, complex treatment changes or for development of acute complications.

Furthermore, the study has a cross-sectional design and thus, knowing that the development of DM complications is a long-term process, it is not possible to establish a timedependent association between UA levels and stroke, UA levels and diabetic retinopathy or UA levels and the evolution of HbAlc. 


\section{Relevance Of The Findings}

HUA is a frequent condition in patients with DM and it is associated with multiple CV risk factors and non-communicable diseases. The results of our study highlight the importance of assessing the UA levels in patients with other cardiometabolic risk factors, with or without DM, for the reason that it appears that UA is correlated with an increased risk of developing $\mathrm{CV}$ events - in this case, stroke, and that it is strongly correlated with an increased body weight, hypertriglyceridemia and chronic kidney disease, all of the above commonly found in diabetic patients.

\section{Future Perspectives}

We aim to further develop this study into a prospective one in which we will evaluate the impact of high levels of UA on the development of DM complications. In addition, a possible future approach would be to include patients with a normal glucose metabolism and with prediabetes in order to assess the risk of DM relying on a history of persistent UA levels. Another perspective could be to include data about the hypouricemic agents and to evaluate their impact on different risk factors, on the glycaemic control and on the complications of DM.

\section{Conclusion}

Serum UA is strongly correlated with the BMI, triglyceride level and GFR in patients with DM. Furthermore, HUA is significantly associated with a higher risk of stroke in these individuals. The resulting cluster of risk factors and complications leads to negative consequences on the global management of DM. Therefore, serum UA should be assessed regularly in diabetic patients and if the levels are high, hypouricemic agents should be prescribed accordingly in order to improve the cardiometabolic profile of the patient.

\section{Disclosure}

The authors report no conflicts of interest in this work.

\section{References}

1. International Diabetes Federation. IDF Diabetes Atlas. 8th ed. Brussels (Belgium): International Diabetes Federation; 2017. Available from: http://www.diabetesatlas.org. Accessed September 24, 2019.

2. Shah AD, Langenberg C, Rapsomaniki E, et al. Type 2 diabetes and incidence of cardiovascular diseases: a cohort study in 1.9 million people Lancet Diabetes Endocrinol. 2015;3(2):105-113. doi:10.1016/S22138587(14)70219-0

3. Singh GM, Danaei G, Farzadfar F, et al. The age-specific quantitative effects of metabolic risk factors on cardiovascular diseases and diabetes: a pooled analysis. PLoS One. 2013;8(7):e65174. doi:10.1371/ journal.pone. 0065174
4. Kanbay M, Jensen T, Solak Y, et al. Uric acid in metabolic syndrome: from an innocent bystander to a central player. Eur J Intern Med. 2016;29:3-8. doi:10.1016/j.ejim.2015.11.026

5. Tuven B, Soysal P, Unutmaz G, Kaya D, Isik AT. Uric acid may be protective against cognitive impairment in older adults, but only in those without cardiovascular risk factors. Exp Gerontol. 2017;89:1519. doi:10.1016/j.exger.2017.01.002

6. Muka T, de Jonge EAL, Kiefte de Jong JC, et al. The influence of serum uric acid on bone mineral density, hip geometry, and fracture risk: the rotterdam study. J Clin Endocrinol Metab. 2016;101 (3):1113-1122. doi:10.1210/jc.2015-2446

7. Krishnan E, Akhras KS, Sharma H, et al. Relative and attributable diabetes risk associated with hyperuricemia in US veterans with gout. QJM. 2013;106(8):721-729. doi:10.1093/qjmed/hct093

8. Bhole V, Choi JWJ, Kim SW, de Vera M, Choi H. Serum uric acid levels and the risk of type 2 diabetes: a prospective study. Am J Med. 2010;123(10):957-961. doi:10.1016/j.amjmed.2010.03.027

9. Juraschek SP, McAdams-Demarco M, Miller ER, et al. Temporal relationship between uric acid concentration and risk of diabetes in a community-based study population. Am J Epidemiol. 2014;179 (6):684-691. doi:10.1093/aje/kwt320

10. Liu J, Tao L, Zhao Z, et al. Two-year changes in hyperuricemia and risk of diabetes: a five-year prospective cohort study. $J$ Diabetes Res. 2018;2018:1-7. doi:10.1155/2018/6905720

11. Serafinceanu C, Elian VI, Catrinoiu D, et al. Clinical and therapeutic characteristics of patients with type 2 diabetes mellitus in Romania MENTOR study. Rom J Diabetes Nutr Metab Dis. 2018;25(4):409418. doi:10.2478/rjdnmd-2018-0049

12. Adiels M, Olofsson S-O, Taskinen M-R, Borén J. Overproduction of very low-density lipoproteins is the hallmark of the dyslipidemia in the metabolic syndrome. Arterioscler Thromb Vasc Biol. 2008;28 (7):1225-1236. doi:10.1161/ATVBAHA.107.160192

13. de Oliveira EP, Burini RC. High plasma uric acid concentration: causes and consequences. Diabetol Metab Syndr. 2012;4(1):12. doi:10.1186/1758-5996-4-12

14. Thounaojam MC, Montemari A, Powell FL, et al. Monosodium urate contributes to retinal inflammation and progression of diabetic retinopathy. Diabetes. 2019;68(5):1014-1025. doi:10.2337/db18-0912

15. American Diabetes Association. 10. cardiovascular disease and risk management: standards of medical care in diabetes - 2019. Diabetes Care. 2019;42(Supplement 1):S103-S123. doi:10.2337/dc19-S010

16. O’Neill S, O’Driscoll L. Metabolic syndrome: a closer look at the growing epidemic and its associated pathologies. Obes Rev. 2015;16 (1):1-12. doi:10.1111/obr.12229

17. Baidog A, Popa AR. Hyperuricemia - as a pathogenetic and independent risk factor in relation to the metabolic syndrome. Rom J Diabetes Nutr Metab Dis. 2018;25(3):335-341. doi:10.2478/rjdnmd-2018-0040

18. Reilly MP, Rader DJ. The metabolic syndrome. Circulation. 2003;108 (13):1546-1551. doi:10.1161/01.CIR.0000088846.10655.E0

19. Zhao J, Chen H, Liu N, et al. Role of hyperhomocysteinemia and hyperuricemia in pathogenesis of Atherosclerosis. J Stroke Cerebrovasc Dis. 2017;26(12):2695-2699. doi:10.1016/j.jstrokec erebrovasdis.2016.10.012

20. Krishnan E, Svendsen K, Neaton JD, Grandits G, Kuller LH; MRFIT Research Group. Long-term cardiovascular mortality among middleaged men with gout. Arch Intern Med. 2008;168(10):1104. doi:10.1001/archinte.168.10.1104

21. Cohen SD, Kimmel PL, Neff R, Agodoa L, Abbott KC. Association of incident gout and mortality in dialysis patients. $J$ Am Soc Nephrol. 2008;19(11):2204-2210. doi:10.1681/ASN.2007111256

22. Choi HK, Curhan G. Independent impact of gout on mortality and risk for coronary heart disease. Circulation. 2007;116(8):894-900. doi:10.1161/CIRCULATIONAHA.107.703389

23. Luk AJ, Levin GP, Moore EE, Zhou X-H, Kestenbaum BR, Choi HK. Allopurinol and mortality in hyperuricaemic patients. Rheumatology (Oxford). 2009;48(7):804-806. doi:10.1093/rheumatology/kep069 
24. Borghi C, Rosei EA, Bardin T, et al. Serum uric acid and the risk of cardiovascular and renal disease. J Hypertens. 2015;33(9):17291741. doi:10.1097/HJH.0000000000000701

25. Cibičková L, Langová K, Vaverková H, Kubíčková V, Karásek D. Correlation of uric acid levels and parameters of metabolic syndrome. Physiol Res. 2017;66(3):481-487.

26. De Cosmo S, Viazzi F, Pacilli A, et al. Serum uric acid and risk of CKD in type 2 diabetes. Clin J Am Soc Nephrol. 2015;10(11):19211929. doi: 10.2215/CJN.03140315

27. Zhong C, Zhong X, Xu T, Xu T, Zhang Y. Sex-specific relationship between serum uric acid and risk of stroke: a dose-response metaanalysis of prospective studies. J Am Heart Assoc. 2017;6(4). doi:10.1161/JAHA.116.005042

28. Du L, Ma J, Zhang X. Higher serum uric acid may contribute to cerebral infarction in patients with type 2 diabetes mellitus: a meta-analysis. $J$ Mol Neurosci. 2017;61(1):25-31. doi:10.1007/s12031-016-0848-y

29. Kawamoto R, Ninomiya D, Kasai Y, et al. Interaction between gender and uric acid on hemoglobin A1c in community-dwelling persons. $J$ Endocrinol Invest. 2018;41(4):421-429. doi:10.1007/s40618-017-0760-5
30. Cui Y, Bu H, Ma X, Zhao S, Li X, Lu S. The relation between serum uric acid and HbA1c is dependent upon hyperinsulinemia in patients with newly diagnosed type 2 diabetes mellitus. $J$ Diabetes Res. 2016;2016:7184123. doi:10.1155/2016/7184123

31. Wei F, Chang B, Yang X, Wang Y, Chen L, Li W-D. Serum uric acid levels were dynamically coupled with hemoglobin A1c in the development of type 2 diabetes. Sci Rep. 2016;6:28549. doi:10.1038/srep28549

32. Singh JA, Yu S. Allopurinol and the risk of stroke in older adults receiving medicare. BMC Neurol. 2016;16(1):164. doi:10.1186/ s12883-016-0692-2

33. Weisman A, Tomlinson GA, Lipscombe LL, Perkins BA, Hawker GA. Association between allopurinol and cardiovascular outcomes and allcause mortality in diabetes: a retrospective, population-based cohort study. Diabetes Obes Metab. 2019;21(6):1322-1329. doi:10.1111/ dom. 13656

34. Zhao Y, Xu L, Tian D, et al. Effects of sodium-glucose co-transporter 2 (SGLT2) inhibitors on serum uric acid level: a meta-analysis of randomized controlled trials. Diabetes Obes Metab. 2018;20(2):458462. doi:10.1111/dom. 13101

Diabetes, Metabolic Syndrome and Obesity: Targets and Therapy

\section{Dovepress}

\section{Publish your work in this journal}

Diabetes, Metabolic Syndrome and Obesity: Targets and Therapy is an international, peer-reviewed open-access journal committed to the rapid publication of the latest laboratory and clinical findings in the fields of diabetes, metabolic syndrome and obesity research. Original research, review, case reports, hypothesis formation, expert opinion and commentaries are all considered for publication. The manuscript management system is completely online and includes a very quick and fair peer-review system, which is all easy to use. Visit http://www.dovepress.com/testimonials.php to read real quotes from published authors.

Submit your manuscript here: https://www.dovepress.com/diabetes-metabolic-syndrome-and-obesity-targets-and-therapy-journal 